\title{
[gw22-e0285] ASSOCIATION STUDY OF THE ANGIOTENSIN- CONVERTING ENZYME (ACE) GENE RS4340 AND RS4343 POLYMORPHISM WITH ATRIAL FIBRILLATION
}

Wang Yazhu, Li Ying, Fan Jinqi, Xu Yanping, Xiao Peilin, Long Yi, Yin YuehuiThe Second Affiliated Hospital Of Chongqing Medical University, Chongqing, China

10.1136/heartjnl-2011-300867.168

Objective To investigate the potential association of angiotensin converting enzyme (ACE) gene polymorphism (rs4340 and rs4343) with atrial fibrillation.

Methods A total of 205 patients were recruited and divided into two groups: the atrial fibrillation group (102 cases) and control group ( 99 cases)in the prospective case-control study. The control group was matched to cases based on age, gender, presence of left ventricular dysfunction, presence of coronary heart disease, presence of diabetes, and presence of primary hypertension. The ACE gene polymorphism (rs4340 and rs4343) was genotyped with polymerase chain reaction-restriction fragment length polymorphism (PCR-RFLP) and gene sequencing approach.

Results The genotype distribution and allele frequencies of rs4340 were not different between the atrial fibrillation groups and control $(p=0.652 ; p=0.599, O R=0.895,95 \%$ CI: 0.591 to 1.354, respectively). However, the genotype distribution and allele frequencies of rs 4343 were different between the atrial fibrillation groups and control $(p<0.001 ; p=0.008 \mathrm{OR}=1.745$, $95 \%$ CI: 1.157 to 2.631 ), compared with the control group, GG+AG genotype distribution of atrial fibrillation group was significantly higher than the AA genotype II/AA genotype frequency of atrial fibrillation group was less than that of control group significantly $(\mathrm{p}=0.001, \mathrm{OR}=0.319,95 \% \mathrm{CI}: 0.162$ to 0.627 ), but II/AG genotype frequency of atrial fibrillation group was more than that of control group $(p=0.031, O R=2.408$, $95 \%$ CI: 1.067 to 5.436 ). There were no statistical difference in left atrial dimension or right atrial dimension in rs 4340 and rs4343 of atrial fibrillation group $(p=0.531, p=0.917 ; p=0.819$, $\mathrm{p}=0.553)$.

Conclusion The genotype distribution and allele frequencies of ACE gene rs 4343 polymorphism were significantly correlated with atrial fibrillation, but not rs $4340 \mathrm{G}$ and II/AG genotype may be risk factors for atrial fibrillation, and II/AA may be a protective factor. 